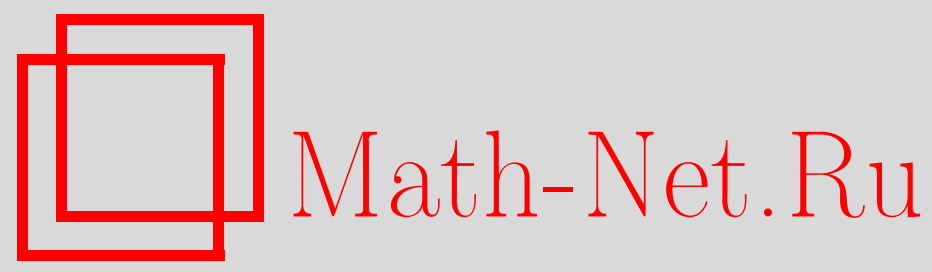

А. Н. Черепов, О приближении непрерывных функций детерминированными функциями с задержкой, Дискрет. матем., 2010, том 22, выпуск 1, 83-103

DOI: https://doi.org/10.4213/dm1086

Использование Общероссийского математического портала Math-Net.Ru подразумевает, что вы прочитали и согласны с пользовательским соглашением http://www . mathnet.ru/rus/agreement

Параметры загрузки:

IP: 52.90 .164 .192

26 апреля 2023 г., $17: 56: 48$ 


\title{
О приближении непрерывных функций детерминированными функциями с задержкой
}

\author{
(ㄱ) 2010 г. А. Н. Черепов
}

\begin{abstract}
Рассматриваются детерминированные функции с задержкой, являющиеся обобщением детерминированных функций, и доказываются некоторые свойства этих функций. Ставится задача приближения непрерывных функций функциями с задержкой и доказано утверждение о возможности приближения с произвольной точностью любой непрерывной функции. Приведены приближения некоторых функций, в том числе функций сложения и умножения, минимальные с точки зрения задержки.
\end{abstract}

\section{1. Введение}

В конце 50-х и начале 60-х годов XX века, А. Н. Колмогоровым и его учениками была рассмотрена задача приближения непрерывных функций детерминированными [4, 5]. Поскольку для заданного $\varepsilon$ не для всякой непрерывной функции существует $\varepsilon$-равная ей детерминированная функция, были предприняты попытки рассмотрения приближений непрерывных функций функциями, близкими к детерминированным. Например, в [4] была рассмотрена модель, так называемых многозвенных автоматов и был получен ряд результатов о приближении непрерывных функций некоторых классов многозвенными автоматами. В 70-х годах группа немецких математиков исследовала вопрос о возможности реализации непрерывных функций детерминированными $[16,17]$. Вместе с тем, представляется естественным исследовать возможность приближения непрерывных функций детерминированными функциями с задержкой. Заметим, что детерминированные функции с задержкой являются разновидностью операторов с предвосхищением [12-15]. Если считать, что автомат - это преобразователь бесконечных входных последовательностей в такие же выходные последовательности, то появление автоматов с задержкой кажется более естественным, чем многозвенных автоматов, в которых рассматриваются конечные входные наборы. Кроме того, замкнутый класс дискретных функций с задержкой интересен сам по себе, как возможное естественное обобщение класса детерминированных функций. Некоторые результаты, связанные с возможностью приближения непрерывных функций детерминированными функциями с задержкой, были получены в [6-11]. В предлагаемой статье содержится обобщения этих результатов и некоторые новые утверждения о сложности приближения непрерывных функций. 


\section{2. Дискретные детерминированные функции с задержкой и их свойства}

Рассмотрим множество $E$ всех бесконечных двоичных последовательностей. Множество всех функций вида $f: E^{n} \rightarrow E$ будем обозначать $P$. Предположим, что $a_{1}, a_{2}, \ldots, a_{n}$ - произвольные последовательности из $E$, тогда $\tilde{a}=\left(a_{1}, a_{2}, \ldots, a_{n}\right)$ - набор таких последовательностей. Пусть $a_{1}\left|k, a_{2}\right| k, \ldots, a_{n} \mid k$ - первые $k$ членов последовательностей $a_{1}, a_{2}, \ldots, a_{n}$ соответственно, тогда $\tilde{a} \mid k=\left(a_{1}\left|k, a_{2}\right| k, \ldots, a_{n} \mid k\right)$. Назовем функцию $f$ детерминированной, если для всех $k=1,2, \ldots$

$$
\forall \tilde{a}, \tilde{b}: \quad \tilde{a}|k=\tilde{b}| k \quad \Longrightarrow \quad f(\tilde{a})|k=f(\tilde{b})| k .
$$

Класс всех детерминированных функций обозначим $P_{d}$, все остальные функции из $P$ будем называть недетерминированными.

Заметим, что понятие детерминированной функции может быть интерпретировано следующим образом. Пусть, как и раньше, $\tilde{a}$ есть $n$-местный набор бесконечных последовательностей из $E$ и $F(\tilde{a})=b$. Предположим, что имеется некоторое дискретное устройство с $n$ входами и одним выходом $F$, и этот дискретный преобразователь функционирует во времени следующим образом: на входы в моменты времени $t=1,2,3, \ldots$ подаются входные последовательности $a_{1}=a_{1}(1) a_{1}(2) \ldots, a_{2}=a_{2}(1) a_{2}(2) \ldots$ и $a_{3}=a_{3}(1) a_{3}(2) \ldots$ В эти же моменты времени $t$ на выходе возникает выходная последовательность $b=b(1) b(2) \ldots$, причем $b=F(\tilde{a})$ и значение $b(k)$ зависит только от значений входных последовательностей в моменты времени $t=1,2,3, \ldots, k$. Функция $F$ является детерминированной [2]. Такие дискретные преобразователи называются автоматами, а реализуемые ими функции - автоматными.

В дальнейшем будут проводиться параллели с автоматами при рассмотрении детерминированных функций. Эти функции часто будут трактоваться как результат работы некоторых автоматов во времени. На множестве $P$ обычным образом введем понятия суперпозиции функций, замкнутого класса и полной системы [2].

Определение 1. Функция $f$ является детерминированной функцией с задержкой $\tau$, где $\tau-$ произвольное неотрицательное целое число, если для любого $k=1,2,3, \ldots$ и любых $\tilde{a}, \tilde{b}$

$$
\tilde{a}|k+\tau=\tilde{b}| k+\tau \quad \Longrightarrow \quad f(\tilde{a})|k=f(\tilde{b})| k .
$$

Множество всех функций с задержкой $\tau$ обозначим $P_{d}^{\tau}$. Заметим, что $P_{d}^{0}=P_{d}$.

Множество функций $P_{d}^{\tau}$ можно определить и следующим образом.

Определение 2. Функция $f$ является детерминированной функцией с задержкой $\tau$, где $\tau$ - произвольное неотрицательное целое число, если существует такая детерминированная функция $g$, что для любого $\tilde{a}$ и $b=g(\tilde{a}), b=b(1) b(2) b(3) \ldots$, значение функции $f$ на $\tilde{a}$ равно

$$
f(\tilde{a})=b(\tau+1) b(\tau+2) b(\tau+3) \ldots
$$

Предложение 1. Определения 1 и 2 эквивалентньл.

Доказательство. Пусть $f$ удовлетворяет условию: для любого $k=1,2,3, \ldots$ и любых $\tilde{a}, \tilde{b}$

$$
\tilde{a}|k+\tau=\tilde{b}| k+\tau \quad \Longrightarrow \quad f(\tilde{a})|k=f(\tilde{b})| k .
$$


Определим дискретную функцию $g$ следующим образом: если $f(\tilde{b})=b(1) b(2) \ldots$, то $g(\tilde{b})=00 \ldots 0 b(1) b(2) \ldots$, где в начале последовательности $00 \ldots 0 b(1) b(2) \ldots$ стоят $\tau$ нулей, то есть функция $g$ в течение первых $\tau$ тактов выдает нули, а затем ее значения совпадают со значениями функции $f$ на том же входном наборе. Покажем, что функция $g$ является детерминированной. Предположим, что $\tilde{a}|k=\tilde{b}| k$ и $k \leqslant \tau$, тогда $g(\tilde{a})|k=g(\tilde{b})| k$, так как функция $g$ в течение первых $\tau$ тактов выдает нули. Пусть теперь $k=i+\tau$, где $i>0$. Тогда из условия $\tilde{a}|i+\tau=\tilde{b}| i+\tau$ следует, что $f(\tilde{a})|i=f(\tilde{b})| i$. Значит, 00..0 $f(\tilde{a})|i+\tau=00 \ldots 0 f(\tilde{b})| i+\tau$, где в начале последовательностей $00 \ldots 0 f(\tilde{a})$ и $00 \ldots 0 f(\tilde{b})$ стоят $\tau$ нулей. Но, по определению функции $g$, $g(\tilde{a})=00 \ldots 0 f(\tilde{a}), g(\tilde{b})=00 \ldots 0 f(\tilde{b})$. Таким образом, при любом $k$

$$
\forall \tilde{a}, \tilde{b}: \quad \tilde{a}|k=\tilde{b}| k \quad \Longrightarrow \quad g(\tilde{a})|k=g(\tilde{b})| k .
$$

Отсюда следует, что функция $g$ является детерминированной и, кроме того, для любого $\tilde{a}$ и $b=g(\tilde{a})$

$$
f(\tilde{a})=b(\tau+1) b(\tau+2) b(\tau+3) \ldots
$$

Пусть теперь функция $g$ является детерминированной и для любого $\tilde{a}$ и $b=g(\tilde{a})$, $b=b(1) b(2) b(3) \ldots$

$$
f(\tilde{a})=b(\tau+1) b(\tau+2) b(\tau+3) \ldots
$$

Рассмотрим произвольное целое $k, k \geqslant 1$, и предположим, что $\tilde{a}|k+\tau=\tilde{c}| k+\tau$. В силу детерминированности функции $g$ справедливо равенство $g(\tilde{a})|k+\tau=g(\tilde{c})| k+\tau$. Но тогда, по определению функции $f$, справедливо равенство $f(\tilde{a})|k=f(\tilde{c})| k$. Значит для любого $k=1,2, \ldots$ и любых $\tilde{a}, \tilde{c}$

$$
\tilde{a}|k+\tau=\tilde{c}| k+\tau \quad \Longrightarrow \quad f(\tilde{a})|k=f(\tilde{c})| k
$$

Предложение доказано.

Из определения 2 следует, что функцию $n$ аргументов из множества $P_{d}^{\tau}$ можно трактовать так: берется дискретное детерминированное устройство с $n$ входами, преобразующее бесконечные входные последовательности из нулей и единиц в такую же выходную последовательность, и рассматривается выход этого устройства не с первого момента времени, а с момента времени $\tau+1$. Осуществляемое преобразование и считается функцией множества $P_{d}^{\tau}$. Таким образом, функции с задержкой являются естественным обобщением детерминированных функций.

Проиллюстрировать связь детерминированных функций и функций с задержкой можно через одно из канонических представлений детерминированной функции - представление посредством дерева. Отметим, что дерево любой детерминированной функции естественным образом связывается с соответствующим автоматом: считается, что для пары $a / b$, приписанной $k$-му элементу $i$-го яруса, $b$ - это то, что выдает автомат в момент времени $i$ при подаче на вход последовательности, получающейся последовательным проходом от корня до выбранной вершины, то есть, перемещаясь по дереву детерминированной функции из корня в вершину, соответствующую подаваемой входной последовательности, вторые элементы каждой пары образуют выходную последовательность. Заметим, что при получении согласно определению 2 из детерминированной функции функций с задержкой, как результат могут получаться и детерминированные функции. Рассмотрим следующий пример. 
Пример 1. Пусть своим деревом задана детерминированная функция $f(x)$, которую можно описать так: в первый момент времени эта функция равна 0 , в последующие моменты, если первым был подан 0 , то на каждом последующем такте работы реализуется полученное на предыдущем такте входное значение $a$, если была подана 1 , то реализуется $\bar{a}$. Из этой функции легко получить функцию множества $P_{d}^{1}$ : будем считывать результат, начиная со второго яруса дерева функции. Таким образом, получаем следующую функцию $f_{1}$ : для любой последовательности $x \in E$ получаем, что $f_{1}(0 x)=0 x, f_{1}(1 x)=0 \bar{x}$ (где под $\bar{x}$ понимается поразрядное отрицание входного набора $x$ ). Эта функция детерминирована. Возьмем $\tau=2$, то есть, будем считывать результат, начиная с третьего яруса дерева функции. Тогда получим функцию $f_{2}$, которая выглядит так: для любой последовательности $x \in E$ получаем, что $f_{2}(00 x)=0 x, f_{2}(01 x)=1 x, f_{2}(10 x)=1 \bar{x}, f_{2}(11 x)=0 \bar{x}$. Очевидно, эта функция уже не будет являться детерминированной.

Приведем пример семейства функций $\left\{\Phi_{\tau}(x)\right\}$, где каждая из функций $\Phi_{\tau}(x)$ принадлежит множеству $P_{d}^{\tau}$. Заметим, что это семейство функций будет использовано в дальнейшем.

Пример 2. Пусть $\tau$ - целое неотрицательное число и $x=x(1) x(2) x(3) \ldots$ Тогда $\Phi_{\tau}(x)$ для любого $x$ определяется так:

$$
\Phi_{\tau}(x)=x(\tau+1) x(\tau+2) x(\tau+3) \ldots
$$

Очевидно, что $\Phi_{\tau}(x) \in P_{d}^{\tau}$ и при $\tau=0$ функция $\Phi_{0}(x)$ реализует тождественное преобразование.

Укажем одно из свойств множеств $P_{d}^{\tau}$.

Свойство 1. Если $\tau \leqslant v$, то $P_{d}^{\tau} \subseteq P_{d}^{v}$. В частности, $P_{d} \subseteq P_{d}^{\tau}$ при всех $\tau=1,2, \ldots$ Доказательство. Пусть $f \in P_{d}^{\tau}$ и $\tau \leqslant v$, тогда для любого $i=1,2, \ldots$ и любых $\tilde{a}, \tilde{b}$

$$
\tilde{a}|i+\tau=\tilde{b}| i+\tau \quad \Longrightarrow \quad f(\tilde{a})|i=f(\tilde{b})| i .
$$

В частности, это будет верно и при $i=v-\tau+1, v-\tau+2, v-\tau+3, \ldots$ Возьмем такие значения $i$ и рассмотрим последовательность значений функции в моменты времени $i+\tau, i=1,2, \ldots$ Эта последовательность совпадает с последовательностью значений функции в моменты времени $j+v$ при $j=1,2,3, \ldots$ Но тогда при всех $j=1,2,3, \ldots$ и любых $\tilde{a}, \tilde{b}$

$$
\tilde{a}|j+v=\tilde{b}| j+v \quad \Longrightarrow \quad f(\tilde{a})|i=f(\tilde{b})| i .
$$

В этом случае $i+\tau=j+v$, поэтому $i \geqslant j$ и из $f(\tilde{a})|i=f(\tilde{b})| i$ следует, что $f(\tilde{a})|j=f(\tilde{b})| j$. Свойство доказано.

Множества функций $P_{d}^{\tau}$ не замкнуты относительно суперпозиции, но их объединение является замкнутым классом.

Определение 3. В класс $P_{d}^{\infty}$ включим все функции множеств $P_{d}^{\tau}$ при $\tau=0,1, \ldots$ Таким образом,

$$
P_{d}^{\infty}=\bigcup_{\tau=0}^{\infty} P_{d}^{\tau}
$$

Предложение 2. Класс $P_{d}^{\infty}$ замкнут. 
Доказательство. Рассмотрим произвольные функции $f, f_{1}, f_{2}, \ldots, f_{n}$. Не ограничивая общности, можно считать, что все эти функции имеют одинаковую арность, вводя при необходимости фиктивные переменные. В силу свойства 1 множеств $P^{\tau}$, также можно считать, что все эти функции принадлежат одному множеству $P_{d}^{\tau}$, выбрав в качестве такового наибольшее из множеств, которым принадлежат функции $f, f_{1}, f_{2}, \ldots, f_{n}$. Покажем, что суперпозиция этих функций $f\left(f_{1}, f_{2}, \ldots, f_{n}\right)$ будет функцией с задержкой $2 \tau$, то есть будет принадлежать множеству $P_{d}^{2 \tau}$. Рассмотрим произвольные наборы $\tilde{a}$ и $\tilde{b}$ такие, что $\tilde{a}|i+2 \tau=\tilde{b}| i+2 \tau$ для любого $i=1,2, \ldots$ Тогда

$$
\begin{aligned}
f_{1}(\tilde{a}) \mid i+\tau & =f_{1}(\tilde{b}) \mid i+\tau, \\
f_{2}(\tilde{a}) \mid i+\tau & =f_{2}(\tilde{a}) \mid i+\tau, \\
\vdots & \\
f_{n}(\tilde{a}) \mid i+\tau & =f_{n}(\tilde{b}) \mid i+\tau,
\end{aligned}
$$

так как $f_{1}, f_{2}, \ldots, f_{n} \in P_{d}^{\tau}$. Но функция $f$ тоже принадлежит $P_{d}^{\tau}$. Поэтому

$$
f\left(f_{1}(\tilde{a}), f_{2}(\tilde{a}), \ldots, f_{n}(\tilde{a})\right)\left|i=f\left(f_{1}(\tilde{b}), f_{2}(\tilde{b}), \ldots, f_{n}(\tilde{b})\right)\right| i,
$$

что и доказывает предложение.

Лемма 1. Пусть $\Phi_{1}(x)$ - одна из функиий $\Phi_{\tau}(x)$ примера 2, рассмотренного ранее, при $\tau=1$. Тогда при любом $\tau>1$

$$
\Phi_{\tau}(x)=\Phi_{1}\left(\Phi_{1}\left(\ldots\left(\Phi_{1}(x)\right) \ldots\right)\right)
$$

где в правой части равенства в суперпозиции участвуют $\tau$ функиий $\Phi_{1}(x)$.

Доказательство. Используем индукцию по $\tau$. Так как $\Phi_{1}(x)=x(2) x(3) \ldots$, при $x=x(1) x(2) x(3) \ldots$ справедливы равенства

$$
\Phi_{1}\left(\Phi_{1}(x)\right)=x(3) x(4) \ldots=\Phi_{2}(x) .
$$

Пусть утверждение доказано для $\tau=k$, докажем его для $\tau=k+1$. Рассмотрим суперпозицию

$$
\Phi(x)=\Phi_{1}\left(\Phi_{1}\left(\ldots\left(\Phi_{1}(x)\right) \ldots\right)\right)
$$

где в правой части использована $k+1$ функция $\Phi_{1}(x)$. По предположению индукции, выполнены равенства

$$
\Phi(x)=\Phi_{1}\left(\Phi_{1}\left(\ldots\left(\Phi_{1}(x)\right) \ldots\right)\right)=\Phi_{1}\left(\Phi_{k}(x)\right) .
$$

Но при $x=x(1) x(2) x(3) x(4) \ldots$, по определению $\Phi_{k}(x)$,

$$
\Phi_{k}(x)=x(k+1) x(k+2) x(k+3) \ldots
$$

Поэтому

$$
\Phi_{1}\left(\Phi_{k}(x)\right)=x(k+2) x(k+3) \ldots=\Phi_{k+1}(x) .
$$


Теорема 1. Справедливо равенство

$$
P_{d}^{\infty}=\left[P_{d} \cup\left\{\Phi_{1}(x)\right\}\right]
$$

Доказательство. Функция $\Phi_{1}(x)$ принадлежит замкнутому классу $P_{d}^{\infty}$, поэтому достаточно показать, что любую функцию этого класса можно получить с помощью суперпозиций детерминированных функций и функции $\Phi_{1}(x)$. Рассмотрим произвольную функцию $f$, принадлежащую множеству $P_{d}^{\tau}$. Согласно определению 2 множества функций $P_{d}^{\tau}$, существует такая детерминированная функция $g$, что при любом $\tilde{a}$, если $b=g(\tilde{a})$, то $f(\tilde{a})=b(\tau+1) b(\tau+2) b(\tau+3) \ldots$ Но тогда $f(\tilde{a})=\Phi_{\tau}(g(\tilde{a}))$ при любом $\tilde{a}$. По лемме 1 , функцию $\Phi_{\tau}(x)$ можно получить суперпозициями функций $\Phi_{1}(x)$, что и доказывает теорему.

\section{3. Действительные детерминированные функции с задержкой и их свойства}

Каждой двоичной последовательности $a(1) a(2) \ldots a(i) \ldots$ сопоставим число из $[0,1]$, равное $0, a(1) a(2) \ldots a(i) \ldots$ Из двух возможных представлений $0, a(1) a(2) \ldots a(i) 100 \ldots=0$, $a(1) a(2) \ldots a(i) 0111 \ldots$ выберем первое. Для числа 1 берем представление $1=0,111 \ldots$ Числа отрезка $[0,1]$, допускающие два представления, будем называть неправильными или двоично-рациональными, остальные числа будем называть правильными. Аналогичным образом перейдем от наборов $\tilde{a}=\left(a_{1}, a_{2}, \ldots, a_{n}\right) \in E^{n}$ к точкам $n$-мерного куба $[0,1]^{n}$. При этом $n$-мерную точку $\vec{b}=\left(b_{1}, b_{2}, \ldots, b_{n}\right) \in[0,1]^{n}$ будем называть неправильной, если хотя бы одна из ее координат является неправильным числом. В противном случае точка правильная или двоично-рациональная. Заметим, что множество двоично-рациональных чисел отрезка $[0,1]$ есть множество чисел, равных рациональным дробям вида $m / 2^{k}$, где $0 \leqslant m / 2^{k} \leqslant 1, m, k-$ целые неотрицательные числа.

Определение 4. Функцию $\mu:[0,1] \rightarrow E$ определим следующим образом: для любого числа $a \in[0,1]$, где $a=0, a_{1} a_{2} a_{3} \ldots$, значение функции равно $\mu(a)=a_{1} a_{2} a_{3} \ldots$

Определение 5. Для любой бесконечной двоичной последовательности $a=a_{1} a_{2} a_{3} \ldots$ определим функцию $\mu^{-1}: E \rightarrow[0,1]$ так, что

(1) если существует натуральное число $k$ такое, что $a=a_{1} a_{2} \ldots a_{k} 01111 \ldots$, то есть, начиная с k+2 члена, $a$ представляет собой бесконечную последовательность единиц, то $\mu^{-1}(a)=0, a_{1} a_{2} \ldots a_{k} 1000 \ldots$;

(2) для остальных последовательностей $\mu^{-1}(a)=0, a_{1} a_{2} a_{3} \ldots$

Определение 6. Любой дискретной функции $f: E^{n} \rightarrow E$ однозначно сопоставим действительную функцию $g:[0,1]^{n} \rightarrow[0,1]$ таким образом, что для любой $n$-мерной точки $\left(b_{1}, b_{2}, \ldots, b_{n}\right) \in[0,1]^{n}$

$$
g\left(b_{1}, b_{2}, \ldots, b_{n}\right)=\mu^{-1} f\left(\mu\left(b_{1}\right), \mu\left(b_{2}\right), \ldots, \mu\left(b_{n}\right)\right) .
$$

Действительную функцию $g$ будем называть функцией, соответствующей дискретной функции $f$. 


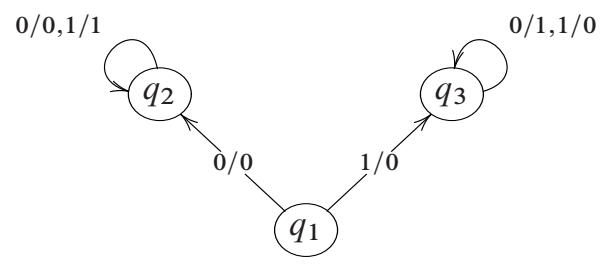

Pис. 1.
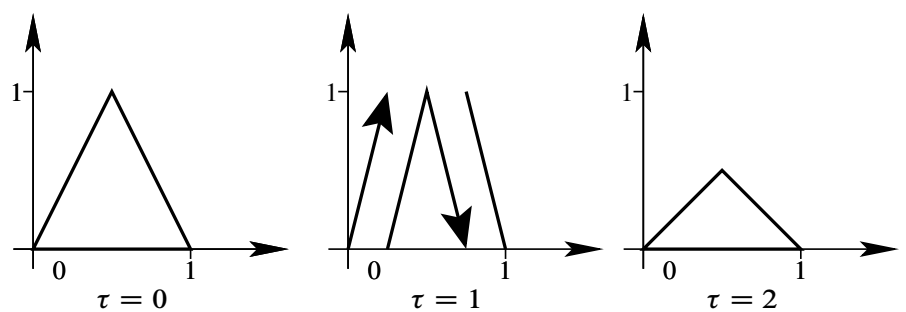

Рис. 2.

Множества действительных функций, соответствующих множествам дискретных функций $P, P_{d}, P_{d}^{\tau}, P_{d}^{\infty}$, будем обозначать $D, \mu^{-1}\left(P_{d}\right), \mu^{-1}\left(P_{d}^{\tau}\right), \mu^{-1}\left(P_{d}^{\infty}\right)$ соответственно. Заметим, что при таком сопоставлении теряется информация о преобразовании последовательностей вида $a_{1} a_{2} \ldots a_{i} 0111 \ldots$ Кроме того, может возникать некоторое несоответствие выхода дискретной функции значению функции действительных аргументов, в том случае, когда выходной последовательности соответствует неправильное (двоично-рациональное) число.

Пример 3. В качестве примера рассмотрим детерминированную функцию, заданную своей диаграммой переходов, и функции действительного переменного, эквивалентные ей в смысле определения 6 , при задержке $\tau=0,1,2$. Рассмотрим функцию с начальным состоянием $q_{1}$, заданную на рис. 1 .

Графики соответствующих функций действительного переменного при $\tau=0,1,2$ имеют вид, показанный на рис. 2.

В дальнейшем более естественным будет рассматривать не сами множества действительных функций $\mu^{-1}\left(P_{d}\right), \mu^{-1}\left(P_{d}^{\tau}\right), \mu^{-1}\left(P_{d}^{\infty}\right)$, а некоторые их подмножества. Это позволит в большей степени отразить содержательный смысл понятия детерминированности.

Определение 7. Пусть $a=0, a_{1} a_{2} a_{3} \ldots$ и $b=0, b_{1} b_{2} b_{3} \ldots$ - действительные числа отрезка $[0,1]$ и $k$ - натуральное число. Тогда будем считать, что $a|k=b| k$, если $\mu(\underset{\sim}{a})|k=\mu(b)| k$, то есть, у чисел совпадают первые $k$ двоичные разрядов. Для точек $\tilde{a}, \tilde{b} \in[0,1]^{n}$ справедливо равенство $\tilde{a}|k=\tilde{b}| k$, если в каждой координате $a_{i}\left|k=b_{i}\right| k$. Действительную функцию $f$ назовем детерминированной, если для любого $k=1,2,3 \ldots$ из равенства $\tilde{a}|k=\tilde{b}| k$ следует, что $f(\tilde{a})|k=f(\tilde{b})| k$. Класс всех детерминированных функций обозначим $D_{d}$. В множество $D_{d}^{\tau}$ действительных функций с задержкой $\tau$ включим все функции $\mu^{-1}\left(P_{d}^{\tau}\right)$, обладающие свойством: для любого $k=1,2,3 \ldots$ из равенства $\tilde{a}|k+\tau=\tilde{b}| k+\tau$ следует, что $f(\tilde{a})|k=f(\tilde{b})| k$. 
Класс $D_{d}^{\infty}$ определим как объединение всех множеств $D_{d}^{\tau}$, то есть

$$
D_{d}^{\infty}=\bigcup_{\tau=0}^{\infty} D_{d}^{\tau} .
$$

Следующий пример показывает, что множества функций $\mu^{-1}\left(P_{d}^{\tau}\right)$ и $D_{d}^{\tau}$ не совпадают.

Пример 4. Укажем дискретную функцию $f(x)$, принадлежащую множеству $P_{d}^{\tau}$, для которой $\mu^{-1} f(\mu(x))$ не принадлежит $D_{d}^{\tau}$. Пусть значение $f(x)$ на всех последовательностях, начинающихся с $0 \ldots 01$, где в начале стоит $\tau+1$ ноль, равно $0111 \ldots$ На остальных последовательностях функция $f(x)$ равна $00 \ldots 0 \ldots$ Тогда

$$
\mu^{-1} f(\mu(0,0 \ldots 0 \ldots))=0,00 \ldots 0 \ldots,
$$

HO

$$
\mu^{-1} f(\mu(0,0 \ldots 0100 \ldots))=0,100 \ldots
$$

(предполагается, что в числе $0,0 \ldots 0100 \ldots$ единица стоит на $\tau+2$ месте). Получаем, что у наборов $\mu(0,0 \ldots 0 \ldots)$ и $\mu(0,0 \ldots 010 \ldots)$ совпадают первые $\tau+1$ значений, но числа $\mu^{-1} f(\mu(00 \ldots 0 \ldots))$ и $\mu^{-1} f(\mu(00 \ldots 0100 \ldots))$ отличаются уже в первом разряде.

Очевидна замкнутость класса $D_{d}$. Нижеследующие доказательства свойства 2 и предложения 3 для функций действительных аргументов по существу повторяют доказательства свойства 1 и предложения 2.

Свойство 2. Если $\tau \leqslant v$, то $D_{d}^{\tau} \subseteq D_{d}^{v}$. В частности, $D_{d} \subseteq D_{d}^{\tau}$ при всех $\tau=1,2, \ldots$ Доказательство. Пусть $f \in D_{d}^{\tau}$ и $\tau \leqslant v$, тогда для любого $i=1,2, \ldots$ и любых $\tilde{a}, \tilde{b} \in[0,1]^{n}$ справедливо свойство

$$
\tilde{a}|i+\tau=\tilde{b}| i+\tau \quad \Longrightarrow \quad f(\tilde{a})|i=f(\tilde{b})| i .
$$

Это свойство будет выполнено и при $i=v-\tau+1, v-\tau+2, v-\tau+3, \ldots$ При таких $i$ последовательность значений $i+\tau$ совпадает с последовательностью значений $j+v$ при $j=1,2,3, \ldots$ Поэтому при всех $j=1,2,3$ и любых $\tilde{a}, \tilde{b}$

$$
\tilde{a}|j+v=\tilde{b}| j+v \quad \Longrightarrow \quad f(\tilde{a})|i=f(\tilde{b})| i,
$$

где $i=v-\tau+j$. Отсюда, поскольку $i \geqslant j$, следует, что

$$
\tilde{a}|j+v=\tilde{b}| j+v \quad \Longrightarrow \quad f(\tilde{a})|i=f(\tilde{b})| i \quad \Longrightarrow \quad f(\tilde{a})|j=f(\tilde{b})| j .
$$

Свойство доказано.

Предложение 3. Класс действительных функиий $D_{d}^{\infty}$ замкнут относительно суперпозиции.

Доказательство. Рассмотрим произвольные функции $f, f_{1}, f_{2}, \ldots, f_{n}$. Не ограничивая общности, можно считать, что все эти функции имеют одинаковую арность, вводя при необходимости фиктивные переменные. В силу свойства 2 множеств $D_{d}^{\tau}$, также можно считать, что все эти функции принадлежат одному множеству, выбрав в качестве такового наибольшее из множеств, которому принадлежат функции $f, f_{1}, f_{2}, \ldots, f_{n}$. Покажем, что суперпозиция этих функций $f\left(f_{1}, f_{2}, \ldots, f_{n}\right)$ будет функцией с задержкой $2 \tau$, то есть 
будет принадлежать множеству $D_{d}^{2 \tau}$. Рассмотрим точки $\tilde{a}$ и $\tilde{b}$ такие, что $\tilde{a}|i+2 \tau=\tilde{b}| i+2 \tau$ при всех $i=1,2, \ldots$ Тогда

$$
\begin{aligned}
f_{1}(\tilde{a}) \mid i+\tau & =f_{1}(\tilde{b}) \mid i+\tau, \\
f_{2}(\tilde{a}) \mid i+\tau & =f_{2}(\tilde{b}) \mid i+\tau, \\
\vdots & \\
f_{n}(\tilde{a}) \mid i+\tau & =f_{n}(\tilde{b}) \mid i+\tau,
\end{aligned}
$$

так как $f_{1}, f_{2}, \ldots, f_{n} \in D_{d}^{\tau}$. Но функция $f$ тоже принадлежит $D_{d}^{\tau}$. Поэтому

$$
f\left(f_{1}(\tilde{a}), f_{2}(\tilde{a}), \ldots, f_{n}(\tilde{a})\right)\left|i=f\left(f_{1}(\tilde{b}), f_{2}(\tilde{b}), \ldots, f_{n}(\tilde{b})\right)\right| i .
$$

Иными словами, показано, что для любого $i=1,2, \ldots$ и любых $\tilde{a}, \tilde{b} \in[0,1]^{n}$

$$
\begin{aligned}
\tilde{a} \mid i+2 \tau & =\tilde{b} \mid i+2 \tau \\
& \Longrightarrow \quad f\left(f_{1}(\tilde{a}), f_{2}(\tilde{a}), \ldots, f_{n}(\tilde{a})\right)\left|i=f\left(f_{1}(\tilde{b}), f_{2}(\tilde{b}), \ldots, f_{n}(\tilde{b})\right)\right| i
\end{aligned}
$$

Предложение доказано.

Аналогом семейства функций $\Phi_{\tau}(x)$ в множествах $D_{d}^{\tau}$ будет семейство функций $F_{\tau}(x)=\left\{2^{\tau} x\right\}$, где $\{a\}-$ дробная часть числа $a$. Для функций $F_{\tau}(x)$ справедливы утверждения, аналогичные утверждениям о функциях $\Phi_{\tau}(x)$.

Лемма 2. При любом $\tau>1$ выполнено равенство

$$
F_{\tau}(x)=F_{1}\left(F_{1}\left(\ldots\left(F_{1}(x)\right) \ldots\right)\right) \text {, }
$$

где в правой части равенства в суперпозиции участвуют $\tau$ функичий $F_{1}(x)$.

Лемма 3. Для любой функици $f$ множества $D_{d}^{\tau}$ сущеествует такая функция $g$ множества $D_{d}$, чтоо $f=F_{\tau}(g)$.

Доказательство. Заметим, что функция $2^{-\tau} x$ является детерминированной, так как при $a=0, a_{1} a_{2} a_{3} \ldots \in[0,1]$

$$
2^{-\tau} a=0,0 \ldots 0 a_{1} a_{2} a_{3} \ldots,
$$

где первые $\tau$ разрядов результата равны 0. Введем обозначение

$$
2^{-\tau} \tilde{x}=\left(2^{-\tau} x_{1}, 2^{-\tau} x_{2}, \ldots, 2^{-\tau} x_{n}\right) .
$$

Пусть функция $f$ принадлежит множеству $D_{d}^{\tau}$. Тогда для любого $i=1,2,3 \ldots$ из $\tilde{a} \mid i+$ $\tau=\tilde{b} \mid i+\tau$ следует, что $f(\tilde{a})|i=f(\tilde{b})| i$. Покажем, что функция $g(\tilde{x})=2^{-\tau} f(\tilde{x})$ также является детерминированной. Если $\tilde{a}|i+\tau=\tilde{b}| i+\tau$ для любого $i=1,2,3 \ldots$, то, учитывая, что умножение на $2^{-\tau}$ сводится к появлению $\tau$ нулей после запятой, получаем, что $2^{-\tau} f(\tilde{a})\left|i+\tau=2^{-\tau} f(\tilde{b})\right| i+\tau$, то есть при $i>\tau$ из $\tilde{a}|i=\tilde{b}| i$ следует равенство $g(\tilde{a})|i=g(\tilde{b})| i$. Поскольку при любом $\tilde{x}$ первые $\tau$ разрядов $g(\tilde{x})$ равны 0, это свойство выполнено и при $i=1,2, \ldots, \tau$. Остается заметить, что справедливо равенство

$$
F_{\tau}(g(\tilde{x}))=F_{\tau}\left(2^{-\tau} f(\tilde{x})\right)=f(\tilde{x}) .
$$

Лемма доказана. 
Из лемм 2 и 3 вытекает следующее утверждение.

Теорема 2. Справедливо равенство

$$
D_{d}^{\infty}=\left[D_{d} \cup\left\{F_{1}(x)\right\}\right]
$$

Докажем некоторые утверждения о непрерывности функций множеств $\mu^{-1}\left(P_{d}\right)$, $\mu^{-1}\left(P_{d}^{\tau}\right), \mu^{-1}\left(P_{d}^{\infty}\right)$.

Предложение 4. Пусть $f \in \mu^{-1}\left(P_{d}^{\tau}\right)$ u $m>\tau$. Тогда если числа $\tilde{a}, \tilde{b}$ совпадают в первых $m$ разрядах, то значения $f(\tilde{a}), f(\tilde{b})$ отличаются не более чем на $2^{-m+\tau}$.

Доказательство. Рассмотрим произвольную функцию $f$, принадлежащую множеству $\mu^{-1}\left(P_{d}^{\tau}\right)$. По определению множества функций $\mu^{-1}\left(P_{d}^{\tau}\right)$ и согласно предложению 1 , существует функция $g \in P_{d}^{\tau}$, соответствующая $f$. Рассмотрим дерево этой функции. Так как значения первых $m$ двоичных разрядов точек $n$-мерного куба $\tilde{a}, \tilde{b}$ совпадают, в дереве им соответствует один и тот же путь длины $m$. Это значит, что, начиная с яруса $\tau+1$ и до яруса $m-\tau$ дерева (эти ярусы определяют первые $m-\tau$ разрядов значения функции $f$ ), значения функции $g$ на этих наборах совпадают, и следовательно, значения функции $f$ различаются менее, чем на $2^{-m+\tau}$, если числа правильные. Возможна ситуация, когда числа различаются в одном из первых $m-\tau$ разрядов. Это может быть, когда одно из чисел $f(\tilde{a}), f(\tilde{b})$ неправильное и ему в дереве функции $g$ соответствует выходная последовательность, которая, начиная с разряда $m-\tau+1$, заканчивается бесконечной последовательностью единиц. Пусть, для определенности это неправильное число равно $f(\tilde{a})$. Обозначим через $f_{m-\tau}$ число, полученное из $f(\tilde{b})$ отбрасыванием последних разрядов, начиная с разряда с номером $(m-\tau+1)$. Тогда, в силу определения функции $\mu^{-1}$, справедливо равенство

$$
f(\tilde{a})=f_{m-\tau}+2^{-m+\tau} .
$$

Значит, и в этом случае разность $|f(\tilde{a})-f(\tilde{b})|$ будет не больше $2^{-m+\tau}$.

Следующие предложения 5, 6 были доказаны Н. Ф. Тюленевым для функций одного аргумента. Докажем их справедливость для функций произвольного числа аргументов.

Предложение 5. Если $f(\tilde{x}) \in \mu^{-1}\left(P_{d}^{\tau}\right)$, то эта функция непрерывна справа во всех точках $\tilde{a} \in[0,1)^{n}$.

Доказательство. Достаточно показать, что для любого $\varepsilon>0$ и для любого значения $\tilde{a} \in[0,1)^{n}$ существует $\tilde{\delta} \in[0,1)^{n}, \delta_{i}>0$ при $i=1, \ldots, n$, такое, что $|f(\tilde{a}+\tilde{\delta})-f(\tilde{a})|<\varepsilon$. Возьмем натуральное число $m$ таким, чтобы $2^{-m}<\varepsilon$. Пусть $\tilde{a}=\left(a_{1}, a_{2}, \ldots, a_{n}\right)$, и пусть $a_{i}=0, a_{1}^{i} a_{2}^{i} \ldots a_{m+\tau}^{i} \ldots(i=1, \ldots, n)$. Тогда для любого $i=1, \ldots, n$ существуют натуральные числа $p_{i}, p_{i}>m+\tau$, такие, что $a_{p_{i}}^{i}=0$ в силу договоренности о представлении чисел отрезка $[0,1]$. Положим $\tilde{\delta}=\left(\delta_{1}, \ldots, \delta_{n}\right)$, где $\delta_{i}=0,0 \ldots 0100 \ldots$ и единица стоит в разряде $p_{i}$. Согласно предложению 4 , значения $f(\tilde{a}+\tilde{\delta})$ и $f(\tilde{a})$ отличаются не более чем на $2^{-m}$. Тем более это справедливо для любого $\tilde{\sigma}$ такого, что $0 \leqslant \sigma_{i}<\delta_{i}$ при $i=1, \ldots, n$, то есть $f(\tilde{a}+\tilde{\sigma})$ и $f(\tilde{a})$ отличаются не более чем на $2^{-m}<\varepsilon$. Предложение 5 доказано.

Предложение 6. Если $f(\tilde{x}) \in \mu^{-1}\left(P_{d}^{\tau}\right)$, то эта функция непрерывна слева во всех правильных точках. 
Доказательство. Аналогично предыдущему случаю, надо показать, что для любого $\varepsilon>0$ и любой правильной точки $\tilde{a}=\left(a_{1}, a_{2}, \ldots, a_{n}\right)$ существует $\delta=\left(\delta_{1}, \delta_{2}, \ldots, \delta_{n}\right), \delta_{i}>0$, $i=1,2, \ldots, n$, такое, что $|f(\tilde{a}-\tilde{\delta})-f(\tilde{a})|<\varepsilon$. Возьмем натуральное число $m$ таким, чтобы $2^{-m}<\varepsilon$. Пусть $a_{i}=0, a_{1}^{i} a_{2}^{i} \ldots$ для всех $i=1, \ldots, n$. Тогда для любого $i$ существуют натуральные числа $p_{i}, p_{i}>m+\tau$, такие, что $a_{p_{i}}^{i}=1$ (так как все $a_{i}$ правильные). Положим $\tilde{\delta}=\left(\delta_{1}, \ldots, \delta_{n}\right)$, где $\delta_{i}=0,0 \ldots 010 \ldots$ и единица стоит в разряде $p_{i}$. Согласно предложению 4, $|f(\tilde{a}-\delta)-f(\tilde{a})|<\varepsilon$. Предложение 6 доказано.

Из предложений 5 и 6 вытекают следующие утверждения.

Следствие 1. Любая функция $f(\tilde{x}) \in \mu^{-1}\left(P_{d}^{\tau}\right)$ непрерывна во всех правильных точках $n$-мерного куба $[0,1]^{n}$.

Следствие 2. Мощность множества точек разрыва любой функиии одного аргумента $f(x)$, где $f \in \mu^{-1}\left(P_{d}^{\tau}\right)$, не более чем счетна.

Доказательство. Точками разрыва функции $f$ могут быть только двоично-рациональные точки, но мощность этого множества счетная.

Поскольку множества $D_{d}, D_{d}^{\tau}, D_{d}^{\infty}$ являются подмножествами соответственно множеств $\mu^{-1}\left(P_{d}\right), \mu^{-1}\left(P_{d}^{\tau}\right), \mu^{-1}\left(P_{d}^{\infty}\right)$, предложения 4-6 и следствия предложений 5,6 справедливы и для них.

Приведем критерий принадлежности функции множеству $D_{d}^{\tau}$. Рассмотрим шаг $h=2^{-k}$ и множество точек отрезка $[0,1]$ вида $x_{i}=i 2^{-k}, i=0,1,2, \ldots, 2^{k}$. В дальнейшем это множество будем называть решеткой точек с шагом $h=2^{-k}$ и обозначать $I_{h}$. Аналогичным образом, в $n$-мерном кубе $[0,1]^{n}$ введем решетку $I_{h}^{n}$, как декартову степень решетки $I_{h}$. Пусть $\tilde{a}=\left(a_{1}, a_{2}, \ldots, a_{n}\right)$ - произвольная точка решетки $I_{h}^{n}$. Интервалом $I_{h}(\tilde{a})$ решетки $I_{h}^{n}$ назовем множество точек $\tilde{x}=\left(x_{1}, x_{2}, \ldots, x_{n}\right) n$-мерного куба $[0,1]^{n}$ таких, что $a_{i} \leqslant x_{i}<h$ при $i=1,2, \ldots$

Теорема 3. Функция $f(\tilde{x})$ принадлежит множеству $D_{d}^{\tau}$ тогда и только тогда, когда

(1) она непрерывна справа во всех точках $n$-мерного куба $[0,1]^{n}$;

(2) при любом $i=\tau+1, \tau+2, \ldots$ на каждом интервале $I_{h}(\tilde{a}), h=2^{-i}$, эта функция удовлетворяет условию

$$
f(\tilde{a} \mid i-\tau) \leqslant f(\tilde{x})<f(\tilde{a} \mid i-\tau)+2^{-(i-\tau)},
$$

где $f(\tilde{a}) \mid i-\tau-$ число, полученное из $f(\tilde{a})$ отбрасыванием всех двоичных разрядов, начиная с разряда $i-\tau+1$.

Доказательство. Докажем необходимость. Любые две точки интервала $I_{h}(\tilde{a}), h=2^{-i}$, совпадают в первых $i$ разрядах по всем координатам. Поэтому, согласно предложению 4, значения функции $f$ на любых точках этого интервала отличаются от значения $f(\tilde{a})$ не более чем на $2^{-(i-\tau)}$. Кроме того, по свойству 2 , если $\tau \leqslant i$, то $D_{d}^{\tau} \subseteq D_{d}^{i}$. Следовательно, значения $f(\tilde{a})$ и $f(\tilde{x}), \tilde{x} \in I_{h}(\tilde{a})$, совпадают в первых $i-\tau$ разрядах.

Докажем достаточность. Пусть выполнены условия теоремы. Для произвольного $i=\tau+1, \tau+2, \ldots$ рассмотрим точки $\tilde{a}, \tilde{b}$ такие, что $\tilde{a}|i=\tilde{b}| i$. Очевидно, что эти точки принадлежат одному интервалу $I_{h}(\tilde{c}), h=2^{-i}$, где $\tilde{c}-$ точка решетки $I_{h}^{n}$, каждая координата которой совпадает в первых $i$ разрядах с соответствующими разрядами координат точек $\tilde{a}, \tilde{b}$. Но тогда, по второму условию теоремы, значения функции $f$ в точках $\tilde{a}, \tilde{b}$ будут совпадать в первых $i-\tau$ разрядах, то есть $f$ принадлежит множеству $D_{d}^{\tau}$. Теорема доказана. 
Пусть $f$ - произвольная функция из $D$ и $I_{h}^{n}, h=2^{-i}, i \geqslant \tau+1$, - некоторая решетка. Обозначим через $f_{h}$ кусочно-постоянную функцию, значения которой на каждом из интервалов $I_{h}(\tilde{a})$ этой решетки равны $f(\tilde{a}) \mid i-\tau$.

Следствие 3. Функиия $f$ принадлежит множеству $D_{d}^{\tau}$ тогда и только тогда, когда при любом $i=\tau+1, \ldots$ на каждом интервале $I_{h}(\tilde{a}), h=2^{-i}$, выполнено неравенство

$$
f_{h} \leqslant f(\tilde{x})<f_{h}+2^{-(i-\tau)} .
$$

Следствие 4. Любая кусочно-константная функиия, значения которой на каждом из интервалов $I_{h}(\tilde{a})$ решетки $I_{h}^{n}, h=2^{-\tau+1}$, постоянны, принадлежит классу $D_{d}^{\tau}$.

Функции множеств $D_{d}, D_{d}^{\tau}, D_{d}^{\infty}$ могут быть разрывными в неправильных точках. Чтобы лучше понять какими свойствами обладают функции этих множеств, выделим в каждом из них подмножества непрерывных функций и обозначим их $C D_{d}, C D_{d}^{\tau}, C D_{d}^{\infty}$ соответственно. Рассмотрим некоторые свойства этих множеств [9].

Для любых точек $\tilde{x}, \tilde{y} \in[0,1]^{n}$ будем считать, что

$$
|\tilde{x}-\tilde{y}|=\max \left|x_{i}-y_{i}\right|,
$$

где максимум берется по всем координатам.

Определение 8. Будем говорить, что функция $f(\tilde{x}) \in D$ удовлетворяет условию Липшица с константой $L$, если для любых $\tilde{x}, \tilde{y}$

$$
|f(\tilde{x})-f(\tilde{y})| \leqslant L|\tilde{x}-\tilde{y}| .
$$

Если для любых $\tilde{x}, \tilde{y}$ выполняется строгое неравенство

$$
|f(\tilde{x})-f(\tilde{y})|<L|\tilde{x}-\tilde{y}|,
$$

то функция строго удовлетворяет условию Липшица. Множество всех функций, удовлетворяющих условию Липшица с константой $L$, обозначим $\operatorname{Lip}(L)$.

Рассмотрим решетку $I_{h}^{n}$. Точки $\tilde{x}$ и $\tilde{y}$ решетки назовем соседними, если либо $\left|x_{i}-y_{i}\right|=h$, либо $\left|x_{i}-y_{i}\right|=0$ для любого $i=1,2, \ldots, n$.

Лемма 4. Пусть $h=2^{-m}, f \in C D_{d}, \tilde{x}, \tilde{y}$-соседние точки в решетке $I_{h}^{n}$, причем либо $x_{i}=y_{i}$, либо $y_{i}=x_{i}+h$ для любого $i=1,2, \ldots, n$. Тогда

$$
|f(\tilde{x})-f(\tilde{y})| \leqslant h .
$$

Доказательство. Рассмотрим последовательность точек $\tilde{z}^{j}$, сходящуюся к $\tilde{y}$. Координаты $\tilde{z}^{j}$ определяются так: если $x_{i}=y_{i}$,то $z_{i}^{j}=x_{i}$ или $z_{i}^{j}=x_{i}+\varepsilon_{i}, 0 \leqslant \varepsilon_{i}<h$, если $y_{i}=x_{i}+h$. Заметим, что при любом $j$ координаты $\tilde{x}, \tilde{z}^{j}$ совпадают в первых $m$ разрядах. Поскольку $f(\tilde{x}) \in C D_{d}$, значения $f(\tilde{x})$ и $f\left(\tilde{z}^{j}\right)$ совпадают в первых $m$ разрядах и

$$
\left|f(\tilde{x})-f\left(\tilde{z}^{j}\right)\right|<2^{-m} .
$$

Переходя к пределу в этом неравенстве и учитывая, что $f$ - непрерывная функция, получаем, что

$$
|f(\tilde{x})-f(\tilde{y})| \leqslant h .
$$


Лемма 5. Пусть $h=2^{-m}, f \in C D_{d}, \tilde{x}, \tilde{y}-$ соседние точки в решетке $I_{h}^{n}$. Тогда

$$
|f(\tilde{x})-f(\tilde{y})| \leqslant 2 h .
$$

Доказательство. Пусть $\tilde{z}=\left(z_{1}, \ldots, z_{n}\right)-$ точка решетки $I_{h}^{n}$, где $z_{i}=\min \left(x_{i}, y_{i}\right)$, $i=1, \ldots, n$. По лемме 4, справедливы неравенства

$$
|f(\tilde{x})-f(\tilde{z})| \leqslant h, \quad|f(\tilde{y})-f(\tilde{z})| \leqslant h .
$$

Отсюда

$$
\begin{aligned}
|f(\tilde{x})-f(\tilde{y})| & =|(f(\tilde{x})-f(\tilde{z}))+(f(\tilde{z})-f(\tilde{y}))| \\
& \leqslant|f(\tilde{x})-f(\tilde{z})|+|f(\tilde{z})-f(\tilde{y})| \leqslant 2 h .
\end{aligned}
$$

Лемма 6. Пусть $h=2^{-m}, f \in C D_{d}, \tilde{x}, \tilde{y}-$ произвольнье точки решетки $I_{h}^{n}$. Тогда

$$
|f(\tilde{x})-f(\tilde{y})| \leqslant 2|\tilde{x}-\tilde{y}| \text {. }
$$

Доказательство. Предположим, что $\max \left|x_{i}-y_{i}\right|=r h$, где $r-$ целое число. Тогда существует последовательность $\tilde{z}^{0}=\tilde{x}, \tilde{z}^{1}, \ldots, \tilde{z}^{r}=\tilde{y}$ из $r+1$ точки решетки $I_{h}^{n}$, в которой любые две последовательные точки являются соседними. Тогда, используя лемму 5, получаем, что

$$
\begin{aligned}
|f(\tilde{x})-f(\tilde{y})| & =\mid\left(f\left(\tilde{z}^{0}\right)-f\left(\tilde{z^{1}}\right)+\left(f\left(\tilde{z}^{1}\right)-f\left(\tilde{z}^{2}\right)\right)+\ldots+\left(f\left(\tilde{z}^{r-1}\right)-f\left(\tilde{z}^{r}\right) \mid\right.\right. \\
& \leqslant\left|f\left(\tilde{z}^{0}\right)-f\left(\tilde{z}^{1}\right)\right|+\left|f\left(\tilde{z^{1}}\right)-f\left(\tilde{z}^{2}\right)\right|+\ldots+\left|f\left(\tilde{z}^{r-1}\right)-f\left(\tilde{z}^{r}\right)\right| \leqslant 2 r h .
\end{aligned}
$$

Лемма доказана.

Теорема 4. Если $f(\tilde{x}) \in C D_{d}$, mo $f(\tilde{x}) \in \operatorname{Lip}(2)$.

Доказательство. Рассмотрим множество $A$ точек всевозможных решеток $I_{h}^{n}$. Заметим, что это множество состоит из всех точек $n$-мерного куба $[0,1]^{n}$, все координаты которых являются двоично-рациональными числами. Пусть $\tilde{x}, \tilde{y} \in A$, тогда существуют такое $h=2^{-m}$ и такая решетка $I_{h}^{n}$, что $\tilde{x}, \tilde{y} \in I_{h}^{n}$. Для любых точек этой решетки по лемме 6 выполнено неравенство

$$
|f(\tilde{x})-f(\tilde{y})| \leqslant 2|\tilde{x}-\tilde{y}| \text {. }
$$

Но множество $A$ всюду плотно в кубе $[0,1]^{n}$ и функция $f$ непрерывна, поэтому это неравенство выполнено и для произвольных точек $\tilde{x}, \tilde{y}$ этого куба.

Следствие 5. Любая функция множества $C D_{d}^{\tau}$ удовлетворяет условию Липшица с константой $2^{\tau+1}$.

Доказательство. Пусть $f(\tilde{x}) \in C D_{d}^{\tau}$, тогда, по лемме 3 , существует такая функция $g$ множества $C D_{d}$, что $f=F_{\tau}(g)$, где $F_{\tau}(x)=\min \left(1,2^{\tau} x\right)$. Поскольку функция $g$ удовлетворяет условию Липшица с константой 2 и функция $F_{\tau}(x)$ удовлетворяет этому условию с константой $2^{\tau}$, функция $f$ удовлетворяет условию Липшица с константой $2^{\tau+1}$. Следствие доказано. 
А. Н. Колмогоровым было доказано, что всякую непрерывную функцию многих переменных, заданную в единичном кубе и принимающую значения из отрезка $[0,1]$, можно представить в виде суперпозиции непрерывных функций одной переменной и функции сложения [1]. Суперпозиция функций, удовлетворяющих условию Липшица, также удовлетворяет условию Липшица для некоторой константы. Кроме того, функции сложения и умножения удовлетворят этому условию. Известно, что не все непрерывные функции, заданные в $n$-мерном единичном кубе и принимающие значения из отрезка $[0,1]$, удовлетворяют условию Липшица. Например, непрерывная функция

$$
f(x)=x \cos \frac{\pi}{2 x}, \quad 0<x \leqslant 1, \quad f(0)=0
$$

не удовлетворяет условию Липшица [3]. Поэтому из теоремы 4 и следствия из нее получаем следующее утверждение.

Теорема 5. Множество $\left[C D_{d}^{\infty} \cup\{x+y\} \cup\{x * y\}\right]$ не содержит все непрерывныле функиии, определенные на единичном кубе и принимающие значения из отрезка [0, 1].

Таким образом, теорему А. Н. Колмогорова нельзя усилить, заменив множество непрерывных функций одного аргумента $C[0,1]$, на множество непрерывных детерминированных функций с задержкой, причем любого числа аргументов, даже если разрешить использовать функцию умножения.

\section{4. Приближение непрерывных функций действительными функциями с задержкой}

Задача приближения непрерывных функций функциями специального вида очень часто появляется в математике. Детерминированные функции являются хорошей моделью реальных устройств - преобразователей сигналов, работающих в дискретные моменты времени. Переход от дискретных функций к функциям вещественных переменных, позволяет ставить вопрос о приближении непрерывных функций функциями с задержкой.

Определение 9. Пусть $\varepsilon>0$, будем говорить, что функция $d(\tilde{x}) \varepsilon$-равна функции $f(\tilde{x})$ на единичном кубе $[0,1]^{n}$, если при любом $\tilde{x} \in[0,1]^{n}$ справедливо неравенство $\mid f(\tilde{x})-$ $d(\tilde{x}) \mid<\varepsilon$. Будем также говорить, что в этом случае $d(\tilde{x}) \varepsilon$-приближает функцию $f(\tilde{x})$.

Пусть $C[0,1]^{n}-$ множество непрерывных на единичном кубе $[0,1]^{n}$ функций, принимающих значения из отрезка $[0,1]$. Оказывается, что не всякую функцию множества $C[0,1]^{n}$ можно $\varepsilon$-приблизить детерминированными функциями класса $D_{d}$ (следствия из предложений 7,8$)$. Но функций класса $D_{d}^{\infty}$ уже достаточно для решения этой задачи. Следующее утверждение было сформулировано в [6] и в [7] доказано для функций класса $\mu^{-1}\left(P_{d}^{\tau}\right)$. Учитывая малую доступность работы [7] приведем свое доказательство этого утверждения для класса $D_{d}^{\infty}$.

Теорема 6. Для любого $\varepsilon>0$ и любой функции $f(\tilde{x}) \in C[0,1]^{n}$ существует число $\tau \geqslant 0$ и функция $d(\tilde{x}) \in D_{d}^{\tau}$ такие, что $d(\tilde{x})$ в-равна $f(\tilde{x})$.

Доказательство. Рассмотрим произвольное $\varepsilon>0$. Так как непрерывная на компакте функция является равномерно непрерывной, существует $\delta>0$ такое, что

$$
|\tilde{x}-\tilde{y}|<\delta \Longrightarrow|f(\tilde{x})-f(\tilde{y})|<\varepsilon / 2 \text {. }
$$


Выберем $\tau$ так, что $2^{-\tau}<\delta$ и рассмотрим решетку $I_{h}^{n}$ при $h=2^{-\tau}$. Для каждой точки $\tilde{a}_{i}, i=0,1, \ldots$, решетки $I_{h}^{n}$ обозначим через $f_{i}$ значение функции $f$ в этой точке с точностью $\lceil\log 1 / \varepsilon\rceil+1$ разрядов, здесь и в дальнейшем $\lceil *\rceil-$ целая часть числа с избытком, а логарифм берется по основанию 2 . Тогда $f\left(\tilde{a}_{i}\right)-f_{i} \leqslant \varepsilon / 2$ и у числа $f_{i}$ по крайней мере, начиная с $\lceil\log 1 / \varepsilon\rceil+2$ разряда, все разряды равны нулю. Построим функцию $d(\tilde{x}) \in D_{d}^{\tau}$, которая $\varepsilon$-приближает функцию $f$. Для этого рассмотрим двоичное дерево высоты $\tau$, к висячим вершинам которого подвешены деревья $n$-арных детерминированных функций. При этом к висячей вершине, соответствующей первым $\tau$ разрядам точки решетки $\tilde{a}_{i}$, подвесим дерево детерминированной функции, реализующей $\lceil\log 1 / \varepsilon\rceil+1$ первых разрядов числа $f_{i}$, а затем выдающей одни нули. Построенное дерево определяет некоторую функцию $g(\tilde{x}) \in P_{d}^{\tau}$. Пусть $d(\tilde{x})-$ функция множества $D_{d}^{\tau}$, соответствующая функции $g(\tilde{x})$. Значение функции $g(\tilde{x})$ при любом $\tilde{x}$ по крайней мере с $\lceil\log 1 / \varepsilon\rceil+2$ разряда представляет собой нулевую последовательность, поэтому $d(\tilde{x})$ принадлежит множеству $D_{d}^{\tau}$. Тогда для любого $\tilde{x} \in[0,1]^{n}$ и точки $\tilde{a}_{i}$ решетки $I_{h}^{n}$, совпадающей с $\tilde{x}$ в первых $\tau$ разрядах,

$$
\begin{aligned}
|f(\tilde{x})-d(\tilde{x})| & =\left|\left(f(\tilde{x})-f\left(\tilde{a}_{i}\right)\right)+\left(f\left(\tilde{a}_{i}\right)-d(\tilde{x})\right)\right| \\
& \left.\leqslant\left|f(\tilde{x})-f\left(\tilde{a}_{i}\right)\right|+\mid f\left(\tilde{a}_{i}\right)\right)-d(\tilde{x}) \mid<\varepsilon,
\end{aligned}
$$

так как $f_{i}=d(\tilde{x}), f\left(\tilde{a}_{i}\right)-f_{i} \leqslant \varepsilon / 2$ и $\left|\tilde{x}-\tilde{a}_{i}\right|<\delta$. Теорема доказана.

Определение 10. Назовем функцию $f(\tilde{x}) \in D_{d}^{\tau}$ ограниченной $m$ разрядами, если существует такое $m$, что $f(\tilde{x})=0, a_{1} a_{2} \ldots a_{m} 000 \ldots$ при любом $\tilde{x} \in[0,1]^{n}$, то есть, при любом $\tilde{x}$ значение функции является двоично-рациональным числом, причем принадлежащим решетке $I_{h}^{n}$, где $h=2^{-m}$.

Следствие 6. Для любого $\varepsilon>0$ и любой функции $f(\tilde{x}) \in C[0,1]^{n}$ существует ичелое число $\tau \geqslant 0$ и ограниченная $\lceil\log 1 / \varepsilon\rceil+1$ разрядами функиия $d(\tilde{x}) \in D_{d}^{\tau}$ такие, что $d(\tilde{x})$ является в-равной $f(\tilde{x})$.

Доказательство этого утверждения следует из доказательства теоремы 5 с учетом того, что деревья, реализующие соответствующие рациональные числа, можно рассматривать до уровня $\lceil\log 1 / \varepsilon\rceil+1$, так как разряды, после стоящего на этом месте, не могут повлиять на точность приближения, и их можно задать равными нулю.

В дальнейшем будем считать, что $\varepsilon=2^{-k}$, где $k-$ натуральное число и $k>1$. Кроме того, если $d(\tilde{x}) \in D_{d}^{\tau}$ и $d(\tilde{x})-$ функция, $\varepsilon$-равная $f(\tilde{x})$, а $g(\tilde{x})$ - дискретная функция, соответствующая $d(\tilde{x})$, то будем говорить, что и $g(\tilde{x}) \varepsilon$-приближает $f(\tilde{x})$.

Теорема 7. Пусть $\varepsilon=2^{-k}, \tau=k+\lceil\log L\rceil-2$, где $\tau=0$ при $k+\lceil\log L\rceil-2 \leqslant 0$. Если функиия $f(\tilde{x})$, принадлежащая множеству $C[0,1]^{n}$, строго удовлетворяет условию Липшица с константой L на каждом из интервалов $I_{h}(\tilde{a})$ решетки $\left.I_{h}^{n}, h=2^{-(\tau+1)}\right)$, или $L$ не является степенью 2 , то сущуествует функиия $d(\tilde{x}) \in D_{d}^{\tau}$, которая $\varepsilon$-приближает функиию $f(\tilde{x})$.

Доказательство. Рассмотрим кусочно-постоянную функцию $d(\tilde{x})$, значения которой на каждом из интервалов $I_{h}(\tilde{a})$ решетки $I_{h}^{n}, h=2^{-(\tau+1)}$, постоянны и равны $\left(f_{\max }+f_{\min }\right) / 2$, где $f_{\max }, f_{\min }-$ максимум и минимум функции $f(\tilde{x})$ на замыкании интервала $I_{h}(\tilde{a})$. По следствию 4, эта функция принадлежит множеству $D_{d}^{\tau}$. Покажем, что для любой точки $\tilde{x}$ интервала $I_{h}(\tilde{a})$ выполнено неравенство $|f(\tilde{x})-d(\tilde{x})|<\varepsilon$. По условию Липшица, на интервале $I_{h}(\tilde{a})$

$$
|f(\tilde{x})-d(\tilde{x})|<(L / 2) 2^{-\tau-1} \leqslant 2^{-k},
$$


если $f(\tilde{x})$ строго удовлетворяет условию Липшица и

$$
|f(\tilde{x})-d(\tilde{x})| \leqslant(L / 2) 2^{-\tau-1}<2^{-k},
$$

если $L$ не есть степень 2.

Определение 11. Для любой непрерывной функции $f$ и любого $\varepsilon>0$ назовем сложностью реализации функции $f$ наименьшее $\tau$ такое, что существует функция $d(\tilde{x})$ множества $D_{d}^{\tau}$, которая $\varepsilon$-равна $f$. Сложность реализации функции $f$ будем обозначать $L(f, \varepsilon)$, а при $\varepsilon=2^{-k}$ будем считать, что $L(f, \varepsilon)=L(f, k)$.

Приведем примеры $\varepsilon$-приближений некоторых непрерывных функций функциями из множества $D_{d}^{\tau}$, стараясь сделать задержку $\tau$ минимальной.

Пример 5. Рассмотрим функцию сложения $x+y$. Для того, чтобы эта функция принимала значения от 0 до 1, рассмотрим ее некоторое ограничение. Будем считать, что

$$
x+y=\min (x+y, 1) .
$$

Предложение 7. Для функции $x+y$ при любом $\varepsilon=2^{-k}$ справедливо равенство

$$
L(x+y, k)=k-1 .
$$

Доказательство. Так как функция $x+y$ строго удовлетворяет условию Липшица с константой $L=2$ на каждом из интервалов $I_{h}(\tilde{a})$ решетки $I_{h}^{n}, h=2^{-k}$, по теореме 7 для нее при любом $\varepsilon=2^{-k}$ существует $\varepsilon$-приближающая функция, принадлежащая множеству $D_{d}^{\tau}$ при $\tau=k-1$.

Докажем, что ни одна из функций множества $D_{d}^{\tau-1}$ не реализует сложение с нужной точностью. Для этого достаточно показать, что знания $k-1$ первых двоичных разрядов чисел $x, y$ недостаточно для определения первого разряда суммы, при вычислении с точностью $\varepsilon=2^{-k}$. Укажем две пары двоичных чисел $x_{1}, y_{1}$ и $x_{2}, y_{2}$, которые совпадают в первых $k-1$ разрядах соответственно, различаясь в $k$-ом разряде. Кроме того, если $\left|\left(x_{1}+y_{1}\right)-a\right|<\varepsilon$ и $\left|\left(x_{2}+y_{2}\right)-b\right|<\varepsilon$, то в числе $a$ первый разряд обязательно равен нулю, а в числе $b$ - единице. Пусть $\delta>0$ - достаточно малое число. Рассмотрим следующие две пары значений аргументов $x_{1}, y_{1}$ и $x_{2}, y_{2}$ :

$$
\begin{array}{ll}
x_{1}=2^{-1}-2^{-k+2}, & y_{1}=0 ; \\
x_{2}=2^{-1}-\delta, & y_{2}=2^{-k+2}-\delta .
\end{array}
$$

Заметим, что у чисел $x_{1}, x_{2}$ и $y_{1}, y_{2}$ первые $k-1$ разрядов совпадают. Тогда

$$
\begin{aligned}
& x_{1}+y_{1}=2^{-1}-2^{-k+2}, \\
& x_{2}+y_{2}=2^{-1}+2^{-k+2}-2 \delta .
\end{aligned}
$$

Отсюда

$$
\left|\left(x_{1}+y_{1}\right)-\left(x_{2}+y_{2}\right)\right|=2 \cdot 2^{-k+2}-2 \delta>\varepsilon .
$$

Кроме того,

$$
\begin{array}{r}
2^{-1}-\left(x_{1}+y_{1}\right)=2^{-k+2}>\varepsilon, \\
\left(x_{2}+y_{2}\right)-2^{-1}=2^{-k+2}-2 \delta>\varepsilon .
\end{array}
$$


Эти неравенства означают, что любое число $a$, приближающее сумму $x_{1}+y_{1}$ с точностью $\varepsilon$, с необходимостью имеет первый разряд равный 0 , а число $b$, приближающее сумму $x_{2}+y_{2}$ с той же точностью, должно иметь первый разряд 1. Таким образом, функции множества $D_{d}^{\tau-1}$ не могут приближать функцию $x+y$ с точностью $\varepsilon$.

Следствие 7. Функциию сложения нельзя приблизить с точностью $\varepsilon=2^{-k}$ функииями класса $D_{d}$ при любом изелом $k>1$.

Пример 6. Рассмотрим функцию умножения $x \cdot y$, где $0 \leqslant x, y \leqslant 1$.

Предложение 8. Для функичи $x \cdot y$ при любом $\varepsilon=2^{-k}$ справедливо равенство

$$
L(x \cdot y, k)=k-1 .
$$

Доказательство. Покажем, что задержки $\tau=k-1$ достаточно для приближения умножения с точностью $\varepsilon$. Так как функция $x \cdot y$ строго удовлетворяет условию Липшица с константой $L=1$, по теореме 7 для нее существует $\varepsilon$-приближающая функция при любом $\varepsilon=2^{-k}$, принадлежащая множеству $D_{d}^{\tau}$ при $\tau=k-1$. Докажем, что ни одна из функций множества $D_{d}^{\tau-1}$ не реализует умножение с нужной точностью. Аналогично случаю функции сложения, покажем, что знания первых $k-1$ разрядов чисел $x, y$ при вычислениях с точностью $\varepsilon=2^{-k}$ недостаточно для определения первого разряда произведения. Возьмем достаточно малое значение $\delta>0$ и рассмотрим следующие пары значений аргументов:

$$
\begin{array}{ll}
x_{1}=1-\delta, & y_{1}=2^{-1}+2^{-k+1}-\delta ; \\
x_{2}=1-2^{-k+1}, & y_{2}=2^{-1} .
\end{array}
$$

Заметим, что в силу договоренности о представлении двоичных чисел у пар чисел $x_{1}, x_{2}$ и $y_{1}, y_{2}$ первые $k-1$ разрядов соответственно совпадают. Тогда

$$
\begin{aligned}
& x_{1} \cdot y_{1}=2^{-1}+2^{-k+1}-\delta 2^{-1}-\delta-\delta 2^{-k+1}+\delta^{2}>2^{-1}+2^{-k}=2^{-1}+\varepsilon \\
& x_{2} \cdot y_{2}=2^{-1}-2^{-k}=2^{-1}-\varepsilon .
\end{aligned}
$$

Таким образом, при вычислении с точностью $\varepsilon=2^{-k}$ в произведении $x_{1} \cdot y_{1}$ первая двоичная цифра результата с необходимостью должна быть равна 1 , а в произведении $x_{2} \cdot y_{2}$ эта цифра равна нулю.

Следствие 8. Функциюю умножения нельзя приблизить с точностью $\varepsilon=2^{-k}$ функциями класса $D_{d}$ при $k>1$.

Пример 7. Рассмотрим функцию $\sqrt[n]{x}$, где $n$ - натуральное число.

Предложение 9. Для функиии $\sqrt[n]{x}$ при любом $\varepsilon=2^{-k}$ справедливо равенство

$$
L(\sqrt[n]{x}, k)=k(n-1) .
$$

Доказательство. Получим нижнюю оценку значения задержки. Предположим, что функция $d(x)$ с задержкой $\tau$ приближает функцию с точностью $\varepsilon=2^{-k}$. Функция $d(x)$ приближает $\sqrt[n]{x}$ при $x=0$ с точностью $\varepsilon=2^{-k}$, поэтому $d(0)<2^{-k}$. Это означает, что число $d(0)$ начинается с $k$ нулей. С другой стороны, значения $k$ первых разрядов функции $d(x)$ для любого $x$, начинающегося с $\tau+k$ нулей, должны быть нулевыми, иначе при $x=0$ требуемая точность не достигается. Рассмотрим число $y=2^{-(\tau+k)}-\delta$, где $\delta$ достаточно 
мало. Отметим, что $\tau+k$ первых разрядов этого числа равны нулю. Поэтому $\tau$ должно быть таким, чтобы выполнялись неравенства

$$
\sqrt[n]{2^{-(\tau+k)}}=\sqrt[n]{y}+\lambda<2^{-k}+\lambda
$$

где $\lambda$ можно сделать как угодно малым. Отсюда

$$
2^{-(\tau+k)} \leqslant 2^{-n k} \quad \Longrightarrow \quad \tau \geqslant k n-k=(n-1) k .
$$

Покажем, что существует функция множества $D_{d}^{\tau}$, которая после задержки $\tau=k(n-1)$ выдает последовательно $k$ первых двоичных разряда числа $\sqrt[n]{x}$ для любого $x \in[0,1]$. Заметим, что на отрезке $[0,1]$ функция $\sqrt[n]{x}$ монотонно возрастает, а производная этой функции монотонно убывает. Рассмотрим решетку $I_{h}^{n}$, в которой $h=2^{-\tau-k-1}=2^{-k n-1}$. Наибольшее изменение функции $\sqrt[n]{x}$ имеет место на отрезке $\left[0,2^{-k n-1}\right]$ и равно $\sqrt[n]{2^{-k n-1}}=2^{-k \sqrt[n]{2^{-1}}}<2^{-k}$. Обозначим через $f_{h}$ кусочно-постоянную функцию, значения которой на каждом из интервалов $I_{h}(\tilde{a})$ решетки равны $f(\tilde{a})$. Эта функция будет $\varepsilon$-равна функции $f(\tilde{x})$ и, по следствию 4 из теоремы 3 , принадлежать множеству $D_{d}^{\tau}$. Утверждение доказано.

Рассмотрим функцию $x^{n}$, где $n-$ натуральное число.

Предложение 10. Для функичи $x^{n}$ при любом $\varepsilon=2^{-k}, k>1$, лююбом натуральном $n$, $n>1$,

$$
L\left(x^{n}, k\right)=\lceil\log n\rceil+k-2 .
$$

Доказательство. Покажем, что задержки $\tau=\lceil\log n\rceil+k-2$ достаточно для приближения $x^{n}$ с любой точностью $\varepsilon=2^{-k}$. Так как эта функция строго удовлетворяет условию Липшица с константой $L=n$ на каждом из интервалов $I_{h}(a)$ решетки $I_{h}, h=2^{-\tau-1}$, по теореме 7 для нее существует $\varepsilon$-приближающая функция при любом $\varepsilon=2^{-k}$, принадлежащая множеству $D_{d}^{\tau}$ при $\tau=\lceil\log n\rceil+k-2$.

Докажем, что ни одна из функций множества $D_{d}^{\tau-1}$ не реализует $x^{n}$ с нужной точностью. Для этого достаточно показать, что знания $\lceil\log n\rceil+k-2$ первых двоичных разрядов числа $x$ недостаточно для определения разряда с номером $k$ результата при вычислении с точностью $\varepsilon=2^{-k}$. Рассмотрим числа $x=1, y=1-2^{\lceil\log n\rceil+k-2}$, и заметим, что в силу договоренности о представлении чисел отрезка $[0,1]$ эти числа совпадают в первых $\lceil\log n\rceil+k-2$ разрядах. Тогда $x^{n}=1$ и

$$
\begin{aligned}
y^{n}=\left(1-2^{-\lceil\log n\rceil-k+2}\right)^{n} & \leqslant 1-n 2^{-\lceil\log n\rceil-k+2}+(n(n-1) / 2)\left(2^{-\lceil\log n\rceil-k+2}\right)^{2} \\
& <1-n 2^{-\lceil\log n\rceil-k+2}+(1 / 2)\left(n 2^{-\lceil\log n\rceil-k+2}\right)^{2} .
\end{aligned}
$$

Поэтому

$$
x^{n}-y^{n}>n 2^{-\lceil\log n\rceil-k+2}+(1 / 2)\left(n 2^{-\lceil\log n\rceil-k+2}\right)^{2} .
$$

Рассмотрим два случая. Пусть $n$ является степенью двух. Тогда для правой части последнего неравенства

$$
n 2^{-\lceil\log n\rceil-k+2}+(1 / 2)\left(n 2^{-\lceil\log n\rceil-k+2}\right)^{2}=2^{-k+2}-(1 / 2)\left(2^{-k+2}\right)^{2}>2^{-k} .
$$

Отсюда следует, что при приближении $x^{n}=1$ с заданной точностью разряд с номером $k$ должен быть равен 1 , а при таком же приближении $y^{n}$ этот разряд должен равняться 0. 
Пусть теперь $n-$ произвольное целое число. Тогда

$$
x^{n}-y^{n}>n 2^{-\lceil\log n\rceil-k+2}+(1 / 2)\left(n 2^{-\lceil\log n\rceil-k+2}\right)^{2} .
$$

Положим

$$
z=n 2^{-\lceil\log n\rceil-k+2}
$$

и заметим, что $2<z \leqslant 4$. Покажем, что при таких $z$ справедливо неравенство

$$
z-(1 / 2) z^{2}>2^{-k}
$$

Действительно, множество решений этого неравенства включает полуинтервал изменения $z$. Предложение доказано.

Определение 12. Под константной функцией с задержкой $\tau$ будем понимать функцию множества $D_{d}^{\tau}$ такую, что соответствующая ей дискретная функция из $P_{d}^{\tau}$ на выходе после задержки $\tau$ выдает последовательность значений, не зависящую от значений входных сигналов, поступающих после задержки.

Следствие 9. При любом $\varepsilon=2^{-k}$ для функций сложения и умножения существуют приближающие константные функции с задержкой $k-1$, ограниченные $k$ разрядами, в-равные им.

Для доказательства следствия достаточно заметить, что приближающие с точностью $\varepsilon$ функции, построенные в первой части доказательства предложений 8,9 , являются константными с задержкой $k-1$. Более того, эти функции ограничены $k$ разрядами.

Предложение 11. Если функция $f(\tilde{x})$, необязательно непрерывная, приближается функцией $d(\tilde{x}) \in D_{d}^{\tau}$ с точностью $\varepsilon=2^{-k}$, то она приближается константной функцией $c$ задержкой не более $\tau+k-1$, ограниченной $k+1$ разрядом.

Доказательство. Рассмотрим дискретную функцию $h(\tilde{x}) \in P_{d}^{\tau}$, соответствующую приближающей функции $d(\tilde{x})$. Двоичное дерево этой функции выглядит так: это полное двоичное дерево высоты $\tau$, соответствующее задержке, к каждой висячей вершине которого подвешено двоичное дерево детерминированной функции. Согласно следствию из теоремы 5 , можно считать, что $h(\tilde{x})$ является $(k+1)$-ограниченной, то есть с момента времени $(\tau+k+2)$, с учетом задержки $\tau$, ее значения равны 0 , независимо от значений входной последовательности. Укажем, как строится двоичное дерево дискретной функции $r(\tilde{x})$, соответствующей искомой константной функции $s(\tilde{x})$. Полное двоичное дерево функции $r(\tilde{x})$ имеет высоту $\tau+k-1$, эта часть дерева соответствует задержке. К каждой висячей вершине этого дерева подвешено двоичное дерево детерминированной функции, выходные значения которой в течение тактов работы с $\tau+k$ по $\tau+2 k+1$, с учетом задержки, совпадают со значениями функции $h(\tilde{x})$ на ветви двоичного дерева, соответствующей этой же висячей вершине, но в дереве функции $r(\tilde{x})$. На остальных тактах работы значения $r(\tilde{x})$ равны 0. Тогда значения $r(\tilde{x})$ совпадают со значениями $h(\tilde{x})$. Поэтому $s(\tilde{x})$ $\varepsilon$-приближает $f(\tilde{x})$ и является константной с задержкой $\tau+k-1$.

Покажем, что существуют функции, для которых приближенная реализация константной функцией с точностью $\varepsilon=2^{-k}$ увеличивает задержку ровно на $k-1$ по сравнению с наилучшей по задержке реализацией. 
Пример 8. Рассмотрим функцию четности. Пусть $f(n, x)$ - функция, которая осуществляет отображение числа $0, x_{1} x_{2} \ldots$ в число $0, y_{1} y_{2} \ldots$ по следующему правилу:

$$
y_{j}=x_{1}+x_{2}+\ldots+x_{k}+\ldots+x_{n+j}(\bmod 2)
$$

для всех $j=1,2,3, \ldots$

Легко построить автомат с задержкой $n$, реализующий дискретную функцию $g(n, x)$, соответствующую действительной функции $f(n, x)$. После задержки $n$ начинает функционировать автомат с двумя состояниями, состоянием $q_{0}$ для четного числа единиц в сумме $x_{1}+x_{2}+\ldots+x_{j}$ и состоянием $q_{1}$ для нечетного числа единиц. Начальным состоянием этого автомата будет $q_{0}$, если сумма $x_{1}+x_{2}+\ldots+x_{n}$ четна, и $q_{1}$, если она нечетна.

Рассмотрим задачу $\varepsilon$-приближения с минимальной задержкой функции $f(n, x)$ функциями класса $D_{d}^{\infty}$, при $\varepsilon=2^{-k}$. Заметим, что, в силу договоренности о представлении чисел отрезка $[0,1]$, функция $f(n, x)$ принадлежит множеству $\mu^{-1}\left(P_{d}^{n}\right)$, но не принадлежит классу $D_{d}^{\infty}$, поэтому постановка такой задачи имеет смысл. Приближающая $f(n, x)$ с точностью $\varepsilon$ функция $d(x)$ множества $D_{d}^{n}$ определяется так: для любого $x$ значение $d(x)$ равно значению $f(n, x)$, в котором после запятой берутся только $k$ первые двоичные разряда, а все остальные разряды полагаются равными нулю. Сделать задержку меньше $n$ нельзя, так как первый разряд значения функции $f(n, x)$ существенно зависит от $(n+1)$-го значения первых разрядов числа $x$. Очевидно, что для любого константного автомата при реализации функции $g(n, x)$ с точностью $\varepsilon=2^{-k}$ требуется задержка $n+k-1$, так как $k$-й разряд выхода автомата определяется всеми $n+k$ разрядами входа.

Автор выражают свою признательность В. А. Буевичу за постановку задачи и существенную помощь, оказанную при окончательном оформлении результатов.

\section{Список литературы}

1. Колмогоров А. Н., О представлении непрерывных функций нескольких переменных в виде суперпозиции непрерывных функций одного переменного. Докл. АН CССР (1957) 114, №5, 953-956.

2. Кудрявцев В. Б., Алешин С. В., Подколзин А. С., Введение в теорию автоматов. Наука, Москва, 1985.

3. Натансон И. П., Теория функциий вещественной переменной. Наука, Москва, 1974.

4. Офман Ю., Об алгоритмической сложности дискретных функций. Докл. АН СССР (1962) 145, №1, 48-51.

5. Офман Ю., О приближенной реализации непрерывных функций на автоматах. Докл. АН СССР (1963) 152, №4, 823-826.

6. Тюленев Н. Ф., Приближение непрерывных функций дискретными. В сб.: Труды семинара по дискретной математике и ее приложениям. Изд-во мех.-мат. ф-та МГУ, Москва, 1997, с. 148151.

7. Тюленев Н. Ф., О приближении непрерывных функций дискретными. В сб.: Конструкции в алгебре и логике. Тверской гос. университет, Тверь, 1990, с. 110-116.

8. Черепов А. Н., Черепов И. А., О представлении недетерминированных функций детерминированными. В сб.: Тез. докл. ХІІІ Международной конф. «Проблемы теоретической кибернетики», 2. Изд-во мех.-мат. ф-та МГУ, Москва, 2002, с. 191.

9. Черепов А. Н., Черепов И. А., О классификации недетерминированных функций. В сб.: Труды семинара по дискретной математике и ее приложениям. Изд-во мех.-мат. ф-та МГУ, Москва, 2004, c. 160-163. 
10. Черепов А. Н., О сложности приближения непрерывных функций недетерминированными функциями с задержкой. В сб.: Материаль IX Международной конф. «Интеллектуальные системы и компьютерные науки», 1 (2). Изд-во мех.-мат. ф-та МГУ, Москва, 2006, с. 307-310.

11. Черепов И. А., О приближении непрерывных функций детерминированными функциями с задержкой. В сб.: Труды семинара по дискретной математике и ее приложениям. Изд-во мех.мат. ф-та МГУ, Москва, 2004, с. 163-166.

12. Трахтенброт Б. А., Об операторах, реализуемых в логических сетях. Докл. АН СССР (1957) 112, №6, 1005-1007.

13. Трахтенброт Б. А., Конечные автоматы и логика одноместных предикатов. Сибирский матем. журнал (1962) 3, №1, 103-131.

14. Трахтенброт Б. А., Барздинь Я. М., Конечные автоматы (поведение и синтез). Наука, Москва, 1970.

15. Burks A. W., Wright J. B., Theory of logical nets. Proc. IRE (1953) 41, 1357-1365.

16. Bernhardt L., Lindner R., Thiele H., Über sequentiell berechenbare reelle Abbildungen. Elektron. Inform.-Verarb. Kybernetik (1971) 7, 317-329.

17. Valk R., Topologische Wortmengen, topologische Automaten, zustandsendliche, stetige Abbildungen. In: Mitteilungen der Gesellschaft für Mathematik und Datenverarbeitung, 19. GMD, Bonn, 1972, pp. 133-135.

Статья поступила 11.06.2008. 